\title{
Spherical Anisotropic Fluid Distribution in General Relativity
}

\author{
A. Sah, Prakash Chandra \\ Department of Mathematics, M B Govt. P G College, Haldwani, India \\ Email: archanasah7@gmail.com,pcfsouk@gmail.com
}

How to cite this paper: Sah, A. and Chandra, P. (2016) Spherical Anisotropic Fluid Distribution in General Relativity. World Journal of Mechanics, 6, 487-504. http://dx.doi.org/10.4236/wjm.2016.612034

Received: October 11, 2016

Accepted: December 2, 2016

Published: December 7, 2016

Copyright $\odot 2016$ by authors and Scientific Research Publishing Inc. This work is licensed under the Creative Commons Attribution International License (CC BY 4.0).

http://creativecommons.org/licenses/by/4.0/

\begin{abstract}
In the present investigation of a spherically symmetric electrically neutral anisotropic static fluid, we present a new solution of the Einstein's general relativistic field equations. The solution shows positive finite central pressures, central density and central red shift. The causality condition is obeyed at the centre. The anisotropy parameter is zero at the center and monotonically increasing toward the surface. The adiabatic index is also increasing towards the surface. All the other physical quantities such as matter-energy density, radial pressure, tangential pressure, velocity of sound and red shift are monotonically decreasing towards the surface. Further by assuming the surface density $\rho=2 \times 10^{17} \mathrm{~kg} \cdot \mathrm{m}^{-3}$, we have constructed a model of massive neutron star with mass $2.95 M_{\odot}$ with radius $18 \mathrm{~km}$ with all degree of suitability.
\end{abstract}

\section{Keywords}

Anisotropic Fluid Ball, Exact Solutions, Einsteins Field Equations, Compact Star, General Relativity

\section{Introduction}

A compact stellar object is formed by an equilibrium state which is reached after condensation and contraction of a massive gas cloud. At this state thermal radiation pressure together with normal fluid pressure balances the gravitational binding energy. Various studies are made for understanding the formation of compact star, its physical properties and internal structure by the solution of Einstein's field equation. Therefore the static isotropic and anisotropic exact solution which describes the compact star is caused to enthusiasts the Researchers to conduct the work in the same field. The study of interior of massive fluid ball can be made by well behaved solution of Einstein's field equation. These equations were solved by Schwarzschild for the interior of the static 
compact stellar object. The first ever two exact solution of Einstein field equation for a compact object in static equilibrium was obtained by Schwarzschild [1] in 1916. The first solution corresponds to the geometry of the space-time exterior to a static perfect fluid ball, while the other solution describes the interior geometry of a fluid sphere of constant energy-density. Tolman [2] has obtained eight different types of exact solutions for static cases. The III solution corresponds to the constant density solution obtained earlier by Schwarzschild. The V and VI solutions correspond to infinite density and infinite pressure at the centre, hence not considered physically viable. Thus only the IV and VII solutions of Tolman are of physical relevance. Despite the non linear character of Einsteins field equations, various exact solutions for static and spherically symmetric metric are available in the related literature.

The search for the exact solutions is of continuous interest to researcher. Buchdahl [3] proposed a famous bound on the mass radius ratio of relativistic fluid spheres which is an important contribution in order to study the stability of the fluid spheres. DelgatyLake [4] studied all the then existing solutions and established that Adler [5], Heintzmann [6], Finch and Skea [7], etc. do not satisfy all the well behaved conditions and also pointed out that only nine solutions are well behaved; out of which seven in curvature coordinates (Tolman, Patwardhan and Vaidya [8], Mehra [9], Kuchowicz [10], Matese and Whitman [11], Durgapals two solutions [12]) and only two solutions (Nariai [13], Goldman [14]) in isotropic coordinates. Ivanov [15] [16], Pant [17], Maurya and Gupta [18], Pant et al. [19] [20] studied the existing well behaved solutions of Einstein field equations in isotropic coordinates. Recently we have found some exact solutions of Einsteins field equations for anisotropic fluid given by Herrera et al. [21] [22], Komathiraj and Maharaj [23], Thirukkanesh and Regel [24], Sunzu et al. [25], Chaisi and Maharaj [26], Maurya and Gupta [27], Gupta and Maurya [28]. Some pioneer work in Relativity is given by Fuloria et al. [29], Whitman and Burch [30], Bonner and Vickers [31], Pant and Negi [32], Herrera and Santo [33] Tikekar [34], Gupta and Kumar [35], Herrera et al. [36] [37] [38] [39], Tewari and Charan [40] [41] [42], Tewari [43], Ivanov [44] [45] [46] [47] [48], Maurya and Gupta [49] and Pant et al. [50] [51].

In this paper we present a new solution in spherically symmetric canonical coordinates which is well behaved. We present a new general solution of Einstein Field Equations and its detailed study, in order to construct a realistic model of compact star. In our present study the paper consists of eight sections. In Section 2 Einstein's field equations in canonical coordinates are given. Expressions of density, anisotropic pressures( radial and transverse pressures), anisotropy parameter and redshift are incorporated in this section. Section 3 consists of boundary conditions for well behaved solutions. A new class of general well behaved solution of Einstein's field equations in canonical coordinates is given in Section 4. This section also includes a particular solution for $n=-\frac{5}{2}$ for constructing a new realistic model for compact star. Section 5 stipulates the properties of this new class of solution of Einstein's field equations. In 
Section 6 the matching conditions of interior metric of the fluid with the Schwarzschild exterior metric are given. For better illustration of our physically accepted solutions, the relevant physical quantities are presented by means of tables and figures in Section 7. Finally, some concluding remarks have been made in Section 8 .

\section{Einstein's Field Equation in Canonical Coordinates}

The Einstein's field equations of general relativity are

$$
R_{\mu v}-\frac{1}{2} R g_{\mu v}=-\frac{8 \pi G}{c^{4}} T_{\mu \nu}
$$

where $T_{\mu \nu}$, the energy momentum tensor for a anisotropic fluid ball is defined as

$$
T_{\mu \nu}=\left(\rho c^{2}+p_{r}\right) u_{\mu} u_{v}-p_{t} g_{\mu v}+\left(p_{r}-p_{t}\right) x_{\mu} x_{v}
$$

where $\rho$ is the proper density, $p_{r}$ and $p_{t}$ are pressures of the fluid in the direction of $u_{\mu}$ (radial pressure)and orthogonal to $u_{\mu}$ (tangential pressure) respectively, $u_{\mu}$ time-like four-velocity vector, $x_{\mu}$ is the unit space like vector in the direction of radial vector and $g_{v}^{\mu}$ metric tensor of space-time.

The interior space-time metric for spherically symmetric fluid distribution is given by

$$
\mathrm{d} s^{2}=-B^{2} \mathrm{~d} r^{2}-r^{2}\left(\mathrm{~d} \theta^{2}+\sin ^{2} \theta \mathrm{d} \phi^{2}\right)+A^{2} \mathrm{~d} t^{2}
$$

where $\mathrm{A}$ and $\mathrm{B}$ are functions of $\mathrm{r}$ only.

In view of the metric (3) and energy momentum tensor (2), the field Equation (1) gives

$$
\begin{gathered}
\frac{8 \pi G}{c^{4}} p_{r}=\frac{1}{B^{2}}\left(\frac{2 A^{\prime}}{r A}+\frac{1}{r^{2}}\right)-\frac{1}{r^{2}} \\
\frac{8 \pi G}{c^{4}} p_{t}=\frac{1}{B^{2}}\left(\frac{A^{\prime \prime}}{A}+\frac{A^{\prime}}{r A}-\frac{A^{\prime} B^{\prime}}{A B}-\frac{B^{\prime}}{r B}\right) \\
\frac{8 \pi G}{c^{2}} \rho=-\frac{1}{B^{2}}\left(\frac{2 B^{\prime}}{r B}-\frac{1}{r^{2}}\right)+\frac{1}{r^{2}} \\
\frac{8 \pi G}{c^{4}}\left(p_{t}-p_{r}\right)=\Delta(r)=\frac{\delta(r)}{B^{2}}
\end{gathered}
$$

where

$$
\delta(r)=\left(\frac{A^{\prime \prime}}{A}-\frac{B^{\prime}}{r B}-\frac{A^{\prime} B^{\prime}}{A B}-\frac{A^{\prime}}{r A}-\frac{1}{r^{2}}\right)+\frac{B^{2}}{r^{2}}
$$

The gravitational redshift of massive spherically symmetric ball is

$$
1+Z=g_{00}^{\frac{-1}{2}}
$$

which gives central $\left(Z_{0}\right)$ and surface $\left(Z_{\Sigma}\right)$ gravitational redshifts

$$
Z_{0}=\frac{C}{A}-1
$$


and

$$
Z_{\Sigma}=\left(1+\frac{r B^{\prime}}{B}\right)^{-1}-1
$$

\section{Boundary Conditions for Well Behaved Solution}

For well behaved nature of the solution in isotropic coordinates, the following conditions should be satisfied (Bonnor-Vickers [31]):

(i) The solution should be free from geometrical and physical singularities. Metric potentials $A$ and $B$ must be non-zero positive finite for free from geometrical singularities while central pressure, central density, should be positive and finite or $\rho_{0}>0$ and $p_{0}>0$ for free from physical singularities.

(ii) The solution should have maximum positive values of pressure and density at the center and monotonically decreasing towards the surface of fluid object i.e.

(a) $\left(\frac{\mathrm{d} p_{r}}{\mathrm{~d} r}\right)_{0}=0$ and $\left(\frac{\mathrm{d}^{2} p_{r}}{\mathrm{~d} r^{2}}\right)_{0}<0$ such that the radial pressure gradient, $\frac{\mathrm{d} p_{r}}{\mathrm{~d} r}$ is negative for $0 \leq r \leq r_{\Sigma}$.

(b) $\left(\frac{\mathrm{d} p_{t}}{\mathrm{~d} r}\right)_{0}=0$ and $\left(\frac{\mathrm{d}^{2} p_{t}}{\mathrm{~d} r^{2}}\right)_{0}<0$ such that the tangential pressure gradient, $\frac{\mathrm{d} p_{t}}{\mathrm{~d} r}$ is negative for $0 \leq r \leq r_{\Sigma}$.

(c) $\left(\frac{\mathrm{d} \rho}{\mathrm{d} r}\right)_{0}=0$ and $\left(\frac{\mathrm{d}^{2} \rho}{\mathrm{d} r^{2}}\right)_{0}<0$ such that the density gradient, $\frac{\mathrm{d} \rho}{\mathrm{d} r}$ is negative for $0 \leq r \leq r_{\Sigma}$.

(iii) The radial pressure must be equal to the tangential pressure at the center i.e. $\left(p_{r}\right)_{0}=\left(p_{t}\right)_{0}$.

(iv) At boundary radial pressure, $p_{r}$ must vanish while tangential pressure, $p_{t}$ may not vanish.

(v) The radial pressure, $p_{r}$, tangential pressure, $p_{t}$ and density $\rho$ should be positive.

(vi) Solution should have positive value of pressure-density ratio which must be less than 1 (weak energy condition) and less than $\frac{1}{3}$ (strong energy condition) throughout within the fluid object and monotonically decreasing as well. (Pant and Negi [32]).

(vii) The condition that the velocity of sound should be less than that of light throughout the model must be satisfied i.e. $0 \leq \sqrt{\frac{\mathrm{d} p_{r}}{c^{2} \mathrm{~d} \rho}}<1$ and $0 \leq \sqrt{\frac{\mathrm{d} p_{t}}{c^{2} \mathrm{~d} \rho}}<1$. The velocity of sound should be monotonically decreasing towards the surface and increasing with the increase of density i.e. $\frac{\mathrm{d}}{\mathrm{d} r}\left(\frac{\mathrm{d} p_{r}}{\mathrm{~d} \rho}\right)<0$ or $\left(\frac{\mathrm{d}^{2} p_{r}}{\mathrm{~d} \rho^{2}}\right)>0$ and $\frac{\mathrm{d}}{\mathrm{d} r}\left(\frac{\mathrm{d} p_{t}}{\mathrm{~d} \rho}\right)<0$ or $\left(\frac{\mathrm{d}^{2} p_{t}}{\mathrm{~d} \rho^{2}}\right)>0$. In this context it is worth mentioning that the equation of state at ultra-high distribution has the property that the sound speed is decreasing 
outwards.

(viii) The anisotropy factor $\Delta$ should be zero at the center and incresing towards the surface of fluid object.

(ix) For realistic matter, adiabatic index $\gamma>1$ i.e. $\frac{p}{\rho}<\frac{\mathrm{d} p}{\mathrm{~d} \rho}$, everywhere within the ball.

(x) The red shift should be positive finite everywhere within and on the fluid sphere, and is monotonically decreasing in nature with the increase of radius from the center $z_{0}$.

(xi) The stability factor $v_{t}^{2}-v_{r}^{2}$ should lie between -1 and 0 throughout the ball.

Under these conditions, we have to assume the one of the gravitational potential component in such a way that the field Equation (1) can be integrated and solution should be well behaved.

\section{New Class of Well Behaved Solution}

We present the following general analytic solution of the field Equations (4) to (7)

$$
\begin{gathered}
A=b+d\left(1-a r^{2}\right)^{n+1} \\
B=\left(1-a r^{2}\right)^{n}
\end{gathered}
$$

The anisotropic pressures, matter-energy density, red shift and anisotropic parameter of fluid ball are given by

$$
\begin{gathered}
\frac{8 \pi G}{c^{4}} p_{r}=\left\{\frac{-4 a(n+1) d}{\left(1-a r^{2}\right)^{n}\left[b+d\left(1-a r^{2}\right)^{n+1}\right]}+\frac{1-\left(1-a r^{2}\right)^{2 n}}{r^{2}\left(1-a r^{2}\right)^{2 n}}\right\} \\
\frac{8 \pi G}{c^{4}} p_{t}=\left\{\frac{-4 a(n+1) d}{\left(1-a r^{2}\right)^{n}\left[b+d\left(1-a r^{2}\right)^{n+1}\right]}+\frac{2 a n}{\left(1-a r^{2}\right)^{2 n+1}}\right\} \\
\frac{8 \pi G}{c^{2}} \rho=\left\{\frac{-4 a n}{\left(1-a r^{2}\right)^{2 n+1}}+\frac{\left(1-a r^{2}\right)^{2 n}-1}{r^{2}\left(1-a r^{2}\right)^{2 n}}\right\} \\
Z=\frac{(c-b)+d\left(1-a r^{2}\right)^{n+1}}{b+d\left(1-a r^{2}\right)^{n+1}} \\
\Delta=\frac{\left(1-a r^{2}\right)^{2 n+1}+(2 n+1) a r^{2}-1}{r^{2}\left(1-a r^{2}\right)^{2 n+1}}
\end{gathered}
$$

Here $a, b$, and $d$ are arbitrary constants.

In order to construct a new relativistic model, we assume $n=\frac{-5}{2}$ then we have 


$$
\begin{gathered}
A=b+d\left(1-a r^{2}\right)^{-\frac{3}{2}} \\
B=\left(1-a r^{2}\right)^{-\frac{5}{2}}
\end{gathered}
$$

The anisotropic pressures, matter-energy density, red shift and anisotropic parameter of fluid ball are given by

$$
\begin{gathered}
\frac{8 \pi G}{c^{4}} p_{r}=a\left\{\frac{6 d\left(1-a r^{2}\right)^{4}}{b\left(1-a r^{2}\right)^{\frac{3}{2}}+d}-a^{4} r^{8}+5 a^{3} r^{6}-10 a^{2} r^{4}+10 a r^{2}-5\right\} \\
\frac{8 \pi G}{c^{4}} p_{t}=a\left\{\frac{6 d\left(1-a r^{2}\right)^{4}}{b\left(1-a r^{2}\right)^{\frac{3}{2}}+d}-5\left(1-a r^{2}\right)^{4}\right\} \\
\frac{8 \pi G}{c^{2}} \rho=a\left(15-50 a r^{2}+70 a^{2} r^{4}-45 a^{3} r^{6}+11 a^{4} r^{8}\right) \\
Z=\frac{(c-b)\left(1-a r^{2}\right)^{\frac{3}{2}}-d}{b\left(1-a r^{2}\right)^{\frac{3}{2}}+d} \\
\Delta=a\left(10 a r^{2}-20 a^{2} r^{4}+15 a^{3} r^{6}-4 a^{4} r^{8}\right)
\end{gathered}
$$

Here $a, b$, and $d$ are arbitrary constants.

In view of Equations (21) and (22) the rate of change of pressures with radial distance from the center $p_{r}^{\prime}$ and $p_{t}^{\prime}$ are given by

$$
\begin{gathered}
\frac{8 \pi G}{c^{4}} p_{r}^{\prime}=-a^{2} r\left[\frac{6 d\left(1-a r^{2}\right)^{3}\left\{5 b\left(\left(1-a r^{2}\right)^{\frac{3}{2}}+8 d\right\}\right.}{\left\{b\left(\left(1-a r^{2}\right)\right)^{\frac{3}{2}}+d\right\}^{2}}-20+40 a r^{2}-30 a^{2} r^{4}+8 a^{3} r^{6}\right] \\
\frac{8 \pi G}{c^{4}} p_{t}^{\prime}=-a^{2} r\left[\frac{6 d\left(1-a r^{2}\right)^{3}\left\{5 b\left(1-a r^{2}\right)^{\frac{3}{2}}+8 d\right\}}{\left\{b\left(1-a r^{2}\right)^{\frac{3}{2}}+d\right\}^{2}}-40\left(1-a r^{2}\right)^{3}\right]
\end{gathered}
$$

In view of Equation (23), (24) and (25) the rate of change of density, red shift and anisotropy parameter with radial distance from the center $\rho^{\prime}, Z^{\prime}$, and $\Delta^{\prime}$ are given by

$$
\begin{gathered}
\frac{8 \pi G}{c^{2}} \rho^{\prime}=-a^{2} r\left(100-280 a r^{2}+270 a^{2} r^{4}-88 a^{3} r^{6}\right) \\
Z^{\prime}=\frac{-3 c a \mathrm{~d} r\left(1-a r^{2}\right)^{\frac{1}{2}}}{\left\{b\left(1-a r^{2}\right)^{\frac{3}{2}}+d\right\}^{2}}
\end{gathered}
$$




$$
\Delta^{\prime}=a^{2} r\left(20-80 a r^{2}+90 a^{2} r^{4}-32 a^{3} r^{6}\right)
$$

\section{Properties of the Solution}

For real values of metric potentials $A$ and $B, a r^{2}<1 \forall r$. Figure 1 shows that the metric potentials $A$ and $B$ are positive at the center which are slightly and monotonically increasing with $r$ for suitable choice of constants $a, b$, and $d$. For the positive central value of $A,(b+d)>0$.

The central value of $p, \rho$ and $Z$ are given as,

$$
\begin{gathered}
\frac{8 \pi G}{c^{4}}\left(p_{r}\right)_{0}=\frac{a(d-5 b)}{(b+d)}=\frac{8 \pi G}{c^{4}}\left(p_{t}\right)_{0} \\
\frac{8 \pi G}{c^{2}} \rho_{0}=15 a \\
Z_{0}=\frac{c-b-d}{b+d}
\end{gathered}
$$

It is clear from Equaiton (31) to Equaiton (33) that for positive central values of physical quantities $p_{0}, \rho_{0}$, and $Z_{0}$ are positive if $a>0, b+d<c$ and $-1<\frac{b}{d}<\frac{1}{5}$. In view of Equaitons (26) to (30), the variation in the pressure, density, red shift and anisotropy parameter with the radial distance from the center of fluid ball are identically zero at the center.

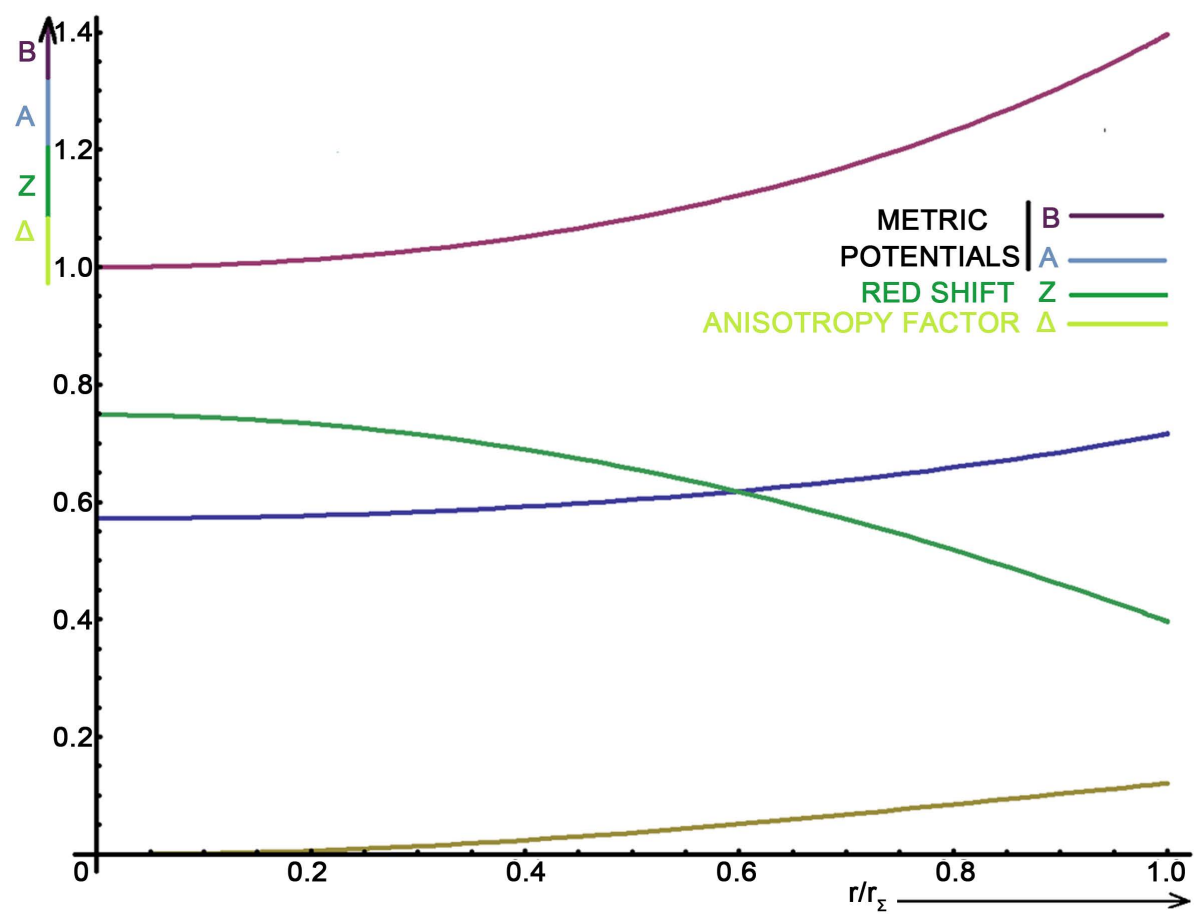

Figure 1. Variation of metric parameters A, B, Red Shift $\mathrm{Z}$ and anisotropy parameter $\Delta$ with $\frac{r}{r_{\Sigma}}$ 
At the center of fluid ball the second order derivatives of radial and tangential pressures with respect to radial distance from the center of fluid ball are

$$
\begin{aligned}
& \frac{8 \pi G}{c^{4}}\left(p_{r}^{\prime \prime}\right)_{0}=-2 a^{2} \frac{\left(14 d^{2}-5 b d-10 b^{2}\right)}{(b+d)^{2}} \\
& \frac{8 \pi G}{c^{4}}\left(p_{t}^{\prime \prime}\right)_{0}=2 a^{2} \frac{\left(-4 d^{2}+25 b d+20 b^{2}\right)}{(b+d)^{2}}
\end{aligned}
$$

The radial pressure is maximum at the center if $\left(p_{r}^{\prime \prime}\right)_{0}<0$ i.e. $\frac{-5-\sqrt{585}}{20}<\frac{b}{d}<\frac{-5+\sqrt{585}}{20}$. The tangential pressure is maximum at the center if $\left(p_{t}^{\prime \prime}\right)_{0}<0$ i.e. $\frac{-25-\sqrt{945}}{40}<\frac{b}{d}<\frac{-25+\sqrt{945}}{40}$. At the center of fluid ball the second order derivative of density with respect to radial distance from the center of fluid ball is

$$
\frac{8 \pi G}{c^{4}}\left(\rho^{\prime \prime}\right)_{0}=-100 a^{2}
$$

The density is maximum at the center for all constants as $\left(\rho^{\prime \prime}\right)_{0}<0$.

The central equation of state

$$
\begin{aligned}
& \left(\frac{p_{r}}{c^{2} \rho}\right)_{0}=\frac{d-5 b}{15(b+d)}=\alpha \\
& \left(\frac{p_{t}}{c^{2} \rho}\right)_{0}=\frac{d-5 b}{15(b+d)}=\beta
\end{aligned}
$$

$\alpha$ and $\beta$ must satisfy the condition $0<\alpha \leq 1$ and $0<\beta \leq 1$ which demands $-\frac{7}{10} \leq \frac{b}{d} \leq \frac{1}{5}$

The central values of square of ratio of speed of sound and speed of light i.e. $\frac{\mathrm{d} p_{r}}{c^{2} \mathrm{~d} \rho}$ and $\frac{\mathrm{d} p_{t}}{c^{2} \mathrm{~d} \rho}$ are given by

$$
\left(\frac{\mathrm{d} p_{\mathrm{r}}}{c^{2} \mathrm{~d} \rho}\right)_{0}=\frac{1}{50} \frac{\left(14 d^{2}-5 b d-10 b^{2}\right)}{(b+d)^{2}}
$$

and

$$
\left(\frac{\mathrm{d} p_{t}}{c^{2} \mathrm{~d} \rho}\right)_{0}=\frac{1}{50} \frac{\left(4 d^{2}-25 b d-20 b^{2}\right)}{(b+d)^{2}}
$$

The causality conditions at the center $0<\left(\frac{\mathrm{d} p_{r}}{c^{2} \mathrm{~d} \rho}\right)_{0} \leq 1$ and $0<\left(\frac{\mathrm{d} p_{t}}{c^{2} \mathrm{~d} \rho}\right)_{0} \leq 1$ give $\frac{-5-\sqrt{585}}{20}<\frac{b}{d}<\frac{-5+\sqrt{585}}{20}$ and $\frac{-25-\sqrt{945}}{40}<\frac{b}{d}<\frac{-25+\sqrt{945}}{40}$ respectively. 
At the center of fluid ball, the second order derivatives of red shift and anisotropy parameter with respect to radial distance from the center of fluid ball are

$$
\begin{gathered}
\left(Z^{\prime \prime}\right)_{0}=-\frac{3 a c d}{(b+d)^{2}} \\
\left(\Delta^{\prime \prime}\right)_{0}=20 a^{2}
\end{gathered}
$$

Red shift is maximum at the center if $\left(Z^{\prime \prime}\right)_{0}<0$ i.e. $d>0$. The anisotropy parameter is minimum at the center since $\left(\Delta^{\prime \prime}\right)_{0}>0$.

It is found that the metric potentials $A$ and $B$ are positive at the center which are slightly and monotonically increasing with $\mathrm{r}$, anisotropy parameter increases from zero at the center to maximum positive value at the boundary and the red shift is zero at the center which is monotonically increasing with $\mathrm{r}$ (Figure 1). The density $\rho$ and pressure $p_{t}$ fall monotonically from their maximum positive values at the center up to non negative values at the boundary while radial pressure $p_{r}$ falls monotonically from it's maximum positive values at the center up to zero value at the boundary (Figure 2) for different values of the parameters $a, b, d$ satisfying

$$
a>0, \quad b<0, \quad d>0, \quad c>b+d>0 \text { and }-\frac{7}{10}<\frac{b}{d}<\frac{-25+\sqrt{945}}{40}
$$

The value of $\alpha=\frac{p_{r}}{c^{2} \rho}$ falls monotonically from it's maximum positive values at the center up to zero value at the boundary and the value of $\beta=\frac{p_{t}}{c^{2} \rho}$ falls monotonically from their maximum positive values at the center up to non negative values at the boundary (Figure 3).

Figure 4 shows that speeds of sound are less than speed of light i.e. $0<\frac{\mathrm{d} p_{r}}{c^{2} \mathrm{~d} \rho} \leq 1$,

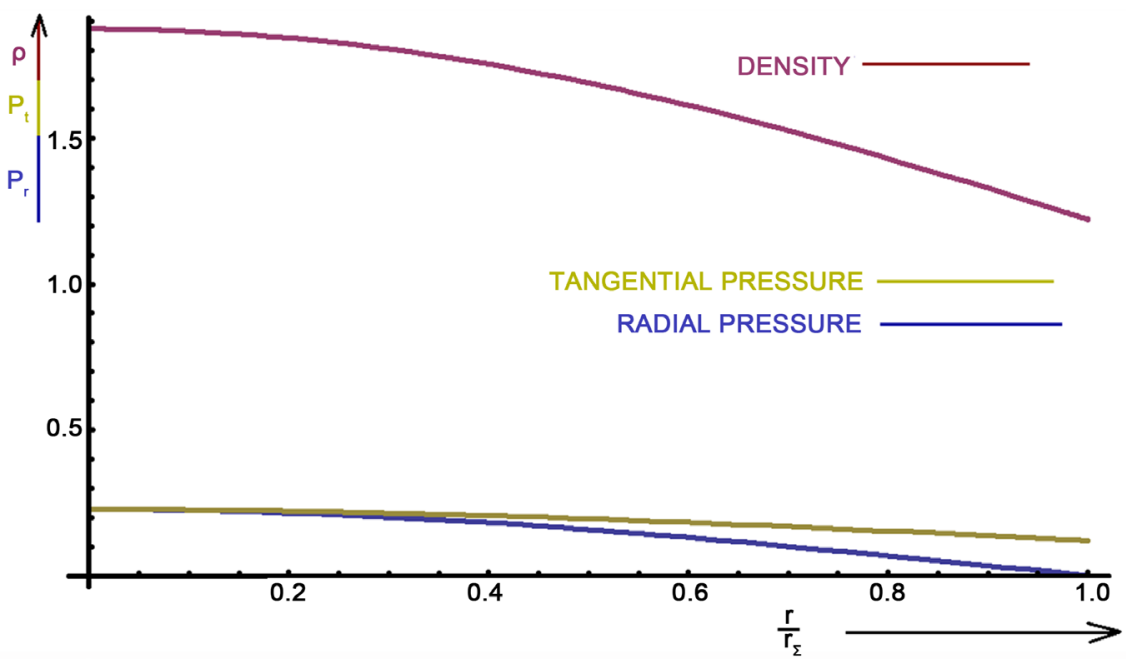

Figure 2. Variation of Density $\rho$, Radial Pressure $p_{r}$ and tangential Pressure $p_{t}$ with $\frac{r}{r_{\Sigma}}$. 


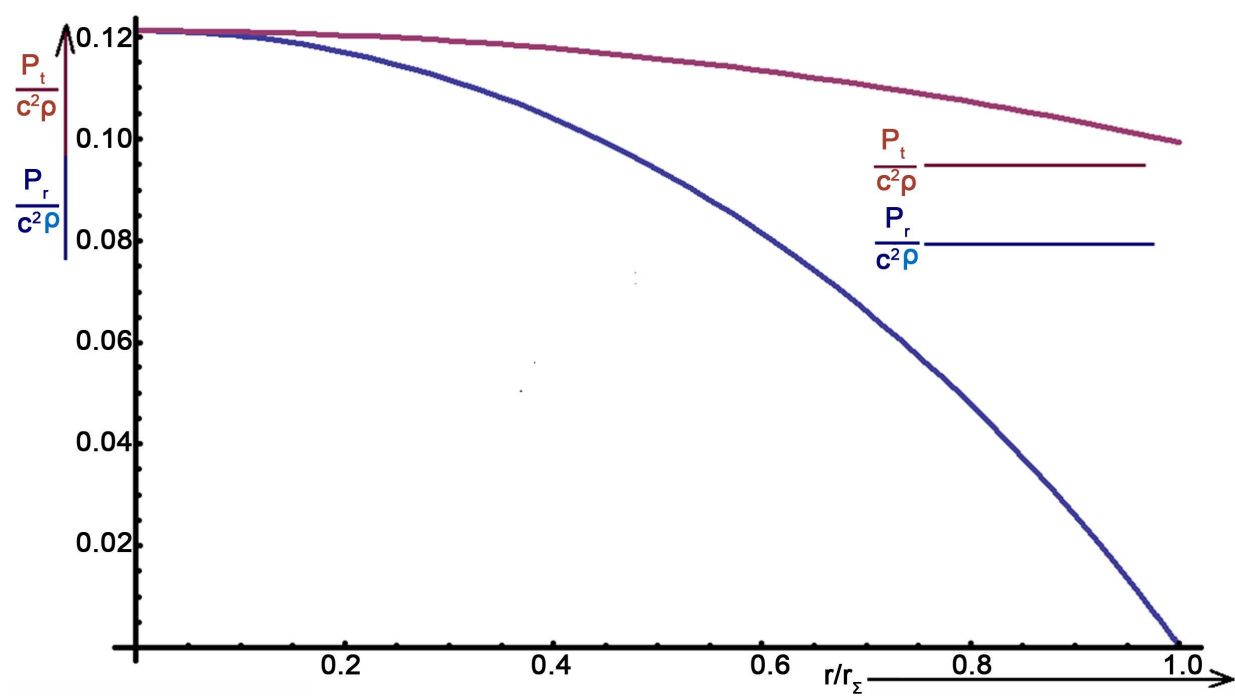

Figure 3. Variation of $\frac{p_{r}}{c^{2} \rho}$ and $\frac{p_{t}}{c^{2} \rho}$ with $\frac{r}{r_{\Sigma}}$.

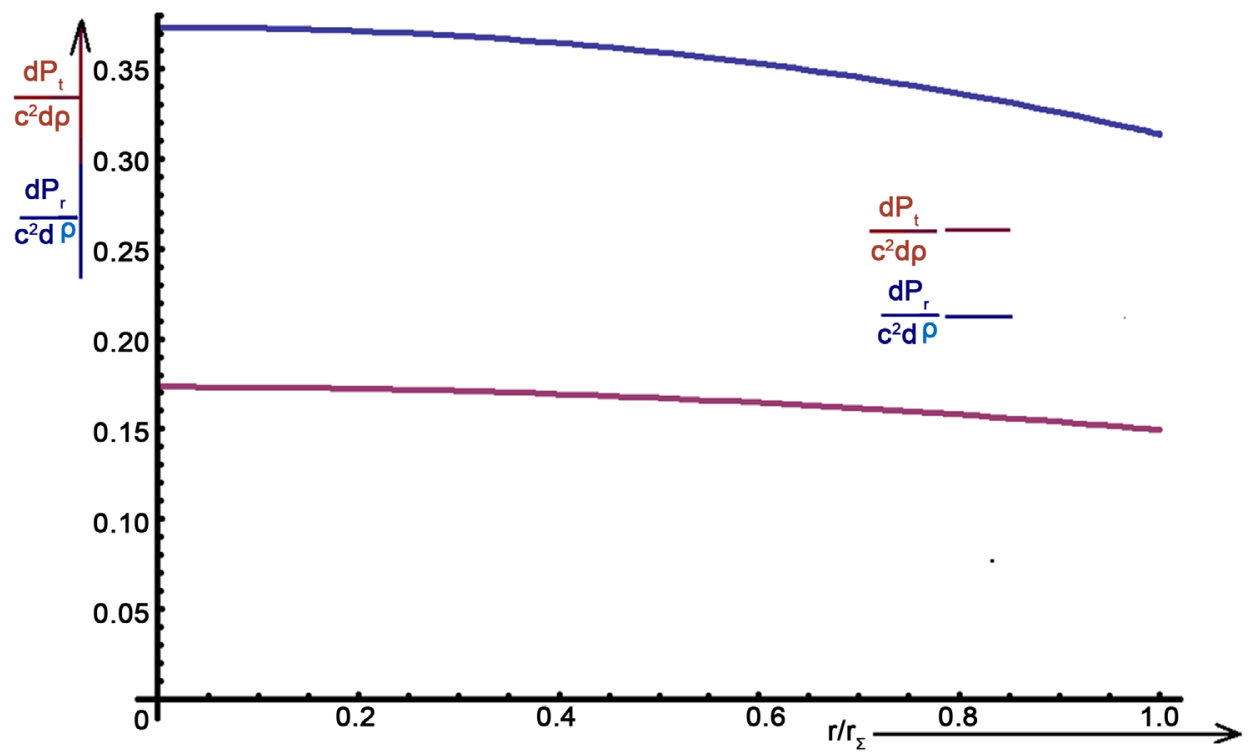

Figure 4. Variation of $\frac{\mathrm{d} p_{r}}{c^{2} \mathrm{~d} \rho}$ and $\frac{\mathrm{d} p_{t}}{c^{2} \mathrm{~d} \rho}$ with $\frac{r}{r_{\Sigma}}$.

$0<\frac{\mathrm{d} p_{t}}{c^{2} \mathrm{~d} \rho} \leq 1$ and the ratio of speeds of sound and light $\sqrt{\frac{\mathrm{d} p}{c^{2} \mathrm{~d} \rho}}$ falls monotonically from center to the boundary of the fluid ball.

The adiabatic index $\gamma\left(\frac{\rho \mathrm{d} p_{r}}{p_{r} \mathrm{~d} \rho}\right.$ and $\left.\frac{\rho \mathrm{d} p_{t}}{p_{t} \mathrm{~d} \rho}\right)$ falls monotonically from their maximum positive values at the center up to non negative values at the boundary (Figure 5).

Figure 6 shows that the stability factor $\left(v_{t}^{2}-v_{r}^{2}\right)$ lies between -1 and 0 . 


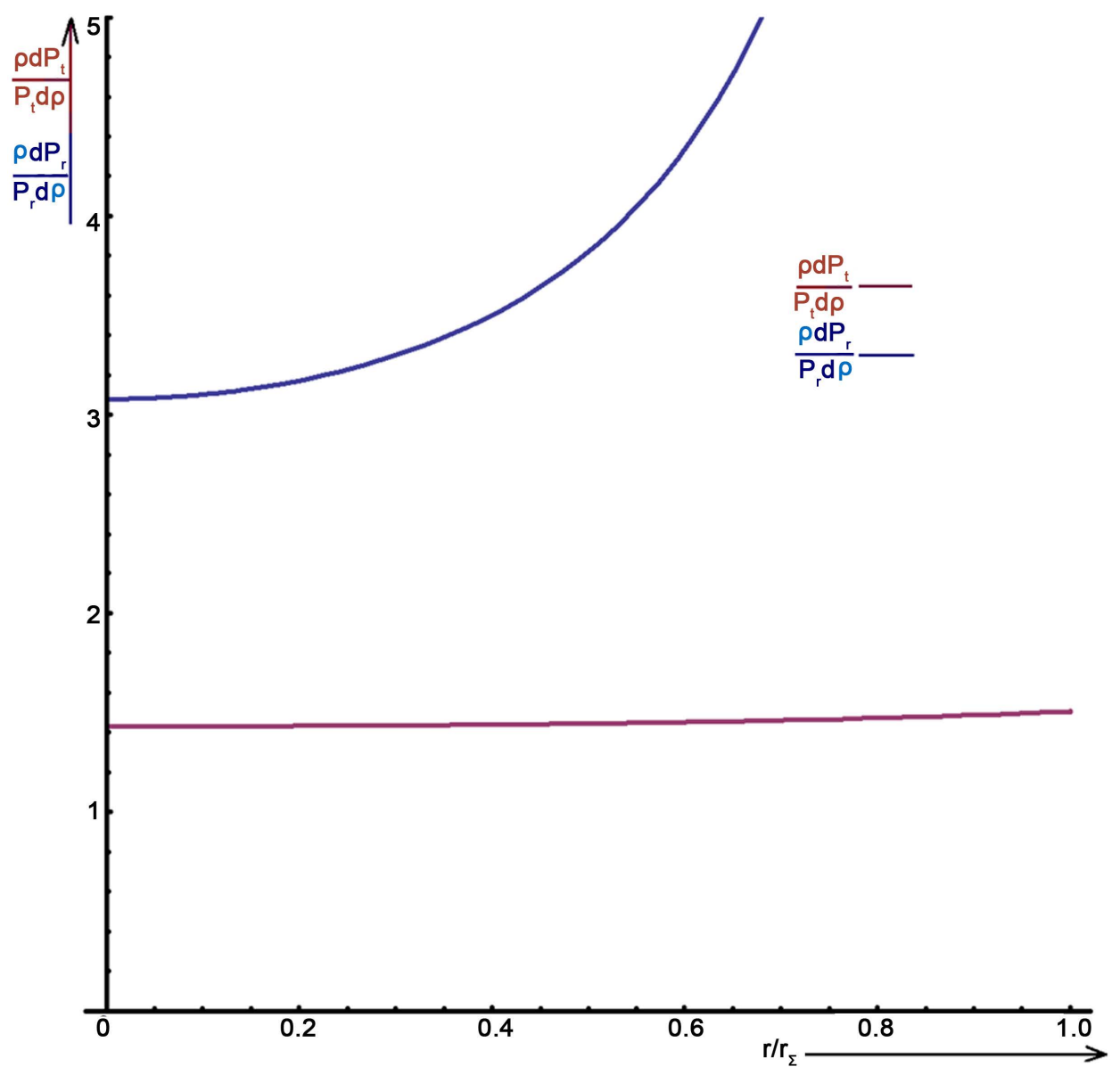

Figure 5. Variation of $\frac{\rho \mathrm{d} p_{r}}{p_{r} \mathrm{~d} \rho}$ and $\frac{\rho \mathrm{d} p_{t}}{p_{t} \mathrm{~d} \rho}$ with $\frac{r}{r_{\Sigma}}$.

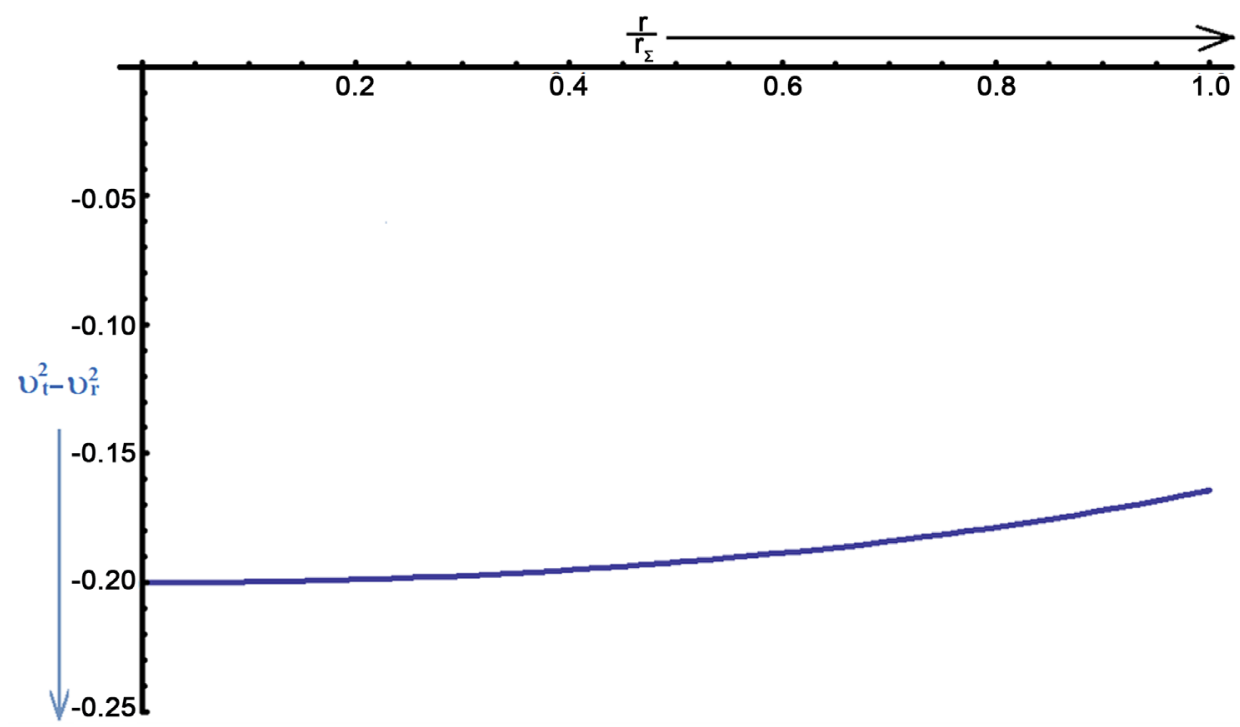

Figure 6. Variation of stability factor $\left(v_{t}^{2}-v_{r}^{2}\right)$ with $\frac{r}{r_{\Sigma}}$. 


\section{Matching Conditions of Boundary}

The solution so obtained are to be matched over the pressure free boundary of fluid sphere smoothly with the Schwarzschild exterior metric:

$$
\mathrm{d} s^{2}=\left(1-\frac{2 G M}{c^{2} r}\right) c^{2} \mathrm{~d} t^{2}-\left(1-\frac{2 G M}{c^{2} r}\right)^{-1} \mathrm{~d} r^{2}-r^{2}\left(\mathrm{~d} \theta^{2}+\sin ^{2} \theta \mathrm{d} \phi^{2}\right)
$$

which requires the continuity of $A^{2}$ and $B^{2}$ across the boundary $r=r_{\Sigma}$ and $\left(p_{r}\right)_{r_{\Sigma}}=0$. Thus

$$
\begin{gathered}
a=\frac{1}{r_{\Sigma}^{2}}\left(1-X^{\frac{1}{5}}\right) ; 0<X<1 \\
b=-\frac{c\left(1+X^{\frac{1}{5}}+X^{\frac{2}{5}}+X^{\frac{3}{5}}-5 X^{\frac{4}{5}}\right)}{6 X^{\frac{3}{10}}} \\
d=\frac{c\left(1+X^{\frac{1}{5}}+X^{\frac{2}{5}}+X^{\frac{3}{5}}+X^{\frac{4}{5}}\right)}{6} \frac{1+X^{\frac{1}{5}}+X^{\frac{2}{5}}+X^{\frac{3}{5}}-5 X^{\frac{4}{5}}}{X^{\frac{3}{10}}\left(1+X^{\frac{1}{5}}+X^{\frac{2}{5}}+X^{\frac{3}{5}}+X^{\frac{4}{5}}\right)}
\end{gathered}
$$

where $X=1-2 S_{p} ; \quad S_{p}=\frac{G M}{c^{2} r_{\Sigma}}$, Schwarzchild parameter.

\section{Tables of Numerical Values of Physical Quantities and Their Graphs}

In view of Equaitons (45) to (48) the values of $X, b, d$ and $\frac{b}{d}$ are $0.4782,-0.078 c, 0.65 c$ and -0.564 respectively and the value of $a r_{\Sigma}^{2}=0.125$ for $S_{p}=0.243$. For better illustration of our physically accepted solution, the relevant physical quantities are presented by means of Table 1 and Table 2 and Figures 1-6 for these constants.

In order to construct a super dense star model, we prescribe the surface density of the star as $2 \times 10^{17} \mathrm{~kg} \cdot \mathrm{m}^{-3}$ and the values of constants $a, b$ and $d$ can be evaluated for different values of $S_{p}$ for describing the well behaved solutions. Thus a compact star model can be constructed by finding mass and radius corresponding to assumed surface density. The variation in the mass and radius with Schwarzschild parameters for our model of compact star is tabulated in the Table 3.

Table 3 shows that neutron star model can be constructed for different values of $S_{p}$ ranging from 0.143 to 0.243 . The variations in $\frac{8 \pi}{c^{2}} G \rho_{\Sigma} r_{\Sigma}^{2}$, radius $R$, mass $M$, central red shift $Z_{0}$ and surface red shift $Z_{\Sigma}$ with Schwarzschild parameter $S_{p}$ are shown in the Figure 7. 


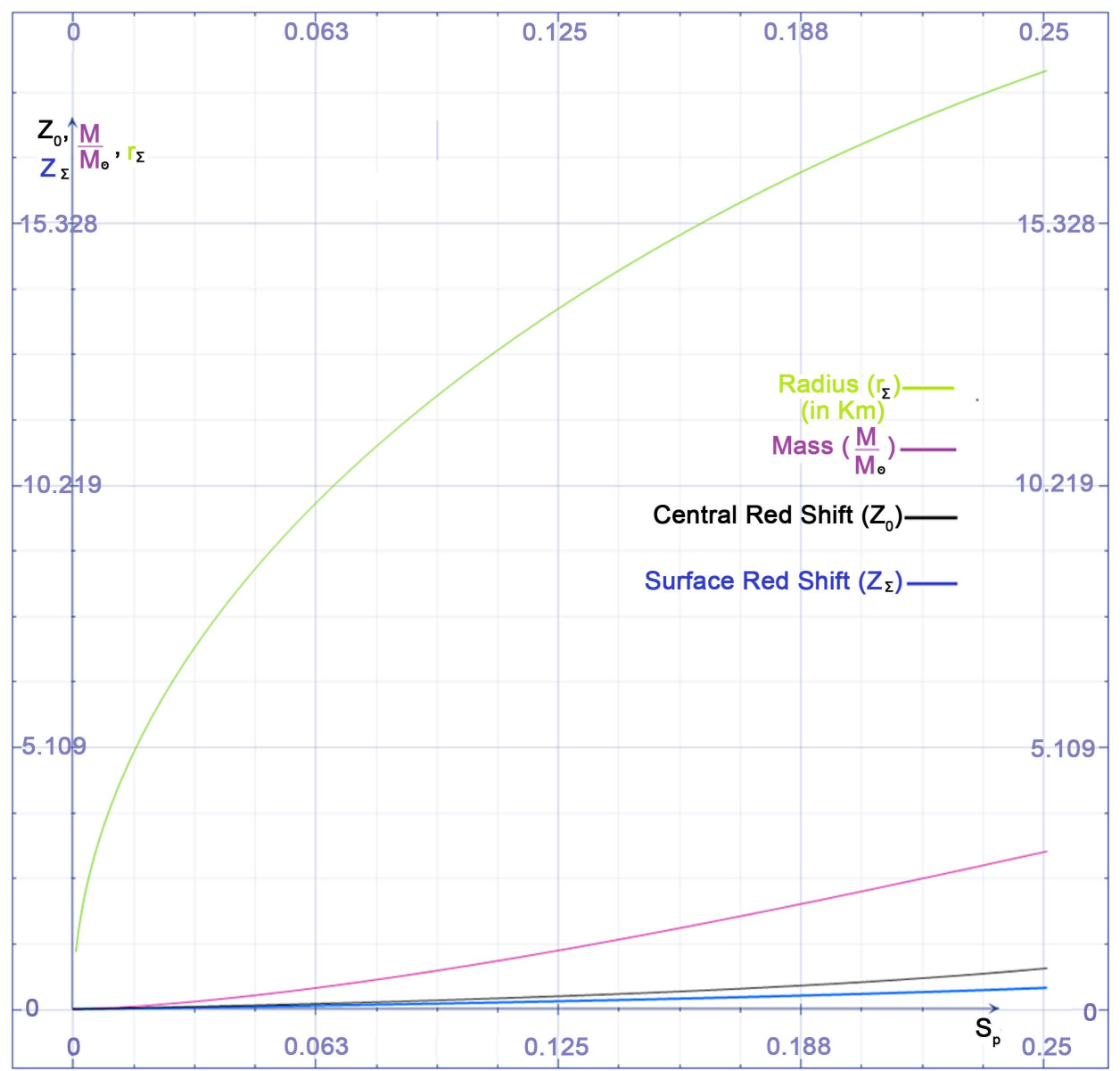

Figure 7. Variation of radius, mass, central red shift and surface red shift with Schwarzschild parameter.

Table 1. $\frac{A}{c}, B, \rho, p_{r}, p_{t}, Z$ and $\Delta$ for $a r_{\Sigma}^{2}=0.125$ or $S_{p}=0.243$.

\begin{tabular}{ccccccccc}
\hline$S . N$. & $\frac{r}{r_{\Sigma}}$ & $\frac{A}{c}$ & $B$ & $\frac{8 \pi G}{c^{2}} \rho r_{\Sigma}^{2}$ & $\frac{8 \pi G}{c^{4}} p_{r} r_{\Sigma}^{2}$ & $\frac{8 \pi G}{c^{4}} p_{t} r_{\Sigma}^{2}$ & $Z$ & $\Delta$ \\
\hline 1 & 0.0 & 0.57200 & 1.00000 & 1.8750 & 0.22727 & 0.22727 & 0.74825 & 0.00000 \\
2 & 0.1 & 0.57322 & 1.00313 & 1.86720 & 0.22436 & 0.22592 & 0.74435 & 0.00156 \\
3 & 0.2 & 0.57690 & 1.01261 & 1.84396 & 0.21572 & 0.22191 & 0.73338 & 0.00680 \\
4 & 0.3 & 0.58312 & 1.02868 & 1.80578 & 0.20160 & 0.21535 & 0.71489 & 0.01375 \\
5 & 0.4 & 0.59200 & 1.05180 & 1.75345 & 0.18243 & 0.20644 & 0.68919 & 0.02401 \\
6 & 0.5 & 0.60370 & 1.08260 & 1.68806 & 0.15876 & 0.19544 & 0.65644 & 0.3667 \\
7 & 0.6 & 0.61848 & 1.12200 & 1.61096 & 0.13129 & 0.18265 & 0.61687 & 0.05135 \\
8 & 0.7 & 0.63664 & 1.17118 & 1.52374 & 0.10083 & 0.16844 & 0.57074 & 0.06761 \\
9 & 0.8 & 0.65860 & 1.23177 & 1.42817 & 0.06824 & 0.15318 & 0.51837 & 0.08494 \\
10 & 0.9 & 0.68487 & 1.30588 & 1.32619 & 0.03446 & 0.13729 & 0.46011 & 0.10283 \\
11 & 1.0 & 0.71614 & 1.39630 & 1.21981 & 0.00000 & 0.12116 & 0.39636 & 0.12103 \\
\hline
\end{tabular}


Table 2. $\frac{p_{r}}{c^{2} \rho}, \frac{p_{t}}{c^{2} \rho}, \frac{d p_{r}}{c^{2} d \rho}$, and $\frac{d p_{t}}{c^{2} d \rho}$ for $a r_{\Sigma}^{2}=0.125$ or $S_{p}=0.243$.

\begin{tabular}{ccccccccc}
\hline$S . N$. & $\frac{r}{r_{\Sigma}}$ & $\frac{p_{r}}{c^{2} \rho}$ & $\frac{p_{t}}{c^{2} \rho}$ & $\frac{\mathrm{d} p_{r}}{c^{2} \mathrm{~d} \rho}$ & $\frac{\mathrm{d} p_{t}}{c^{2} \mathrm{~d} \rho}$ & $\frac{\rho \mathrm{d} p_{r}}{p_{r} \mathrm{~d} \rho}$ & $\frac{\rho \mathrm{d} p_{t}}{p_{t} \mathrm{~d} \rho}$ & $v_{t}^{2}-v_{r}^{2}$ \\
\hline 1 & 0.0 & 0.12121 & 0.12121 & 0.37334 & 0.17334 & 3.08011 & 1.43011 & -0.20000 \\
2 & 0.1 & 0.12016 & 0.12099 & 0.37279 & 0.17309 & 3.10250 & 1.43062 & -0.19970 \\
3 & 0.2 & 0.11698 & 0.12034 & 0.37114 & 0.17235 & 3.17250 & 1.43216 & -0.19879 \\
4 & 0.3 & 0.11164 & 0.11925 & 0.36837 & 0.17111 & 3.29955 & 1.43482 & -0.19726 \\
5 & 0.4 & 0.10404 & 0.11773 & 0.36445 & 0.16938 & 3.50309 & 1.43871 & -0.19507 \\
6 & 0.5 & 0.09405 & 0.11577 & 0.35936 & 0.16272 & 3.82107 & 1.44401 & -0.19664 \\
7 & 0.6 & 0.08150 & 0.11338 & 0.35304 & 0.16451 & 4.331825 & 1.45098 & -0.18853 \\
8 & 0.7 & 0.06617 & 0.11054 & 0.34542 & 0.16138 & 5.22012 & 1.45998 & -0.18404 \\
9 & 0.8 & 0.04778 & 0.10726 & 0.33642 & 0.15782 & 7.04053 & 1.47145 & -0.17860 \\
10 & 0.9 & 0.02598 & 0.10352 & 0.32590 & 0.15383 & 12.54187 & 1.48603 & -0.17207 \\
11 & 1.0 & 0.00000 & 0.09932 & 0.31372 & 0.14943 & $\infty$ & 1.50455 & -0.16429 \\
\hline
\end{tabular}

Table 3. Variation in $\frac{8 \pi G}{c^{2}} \rho_{\Sigma} r_{\Sigma}^{2}, r_{\Sigma}, \rho, \frac{M}{M_{\odot}}, Z_{0}$ and $Z_{\Sigma}$ with Schwarzschild parameter $S_{p}$.

\begin{tabular}{ccccccc}
\hline$S . N$. & $S_{p}$ & $\frac{8 \pi G}{c^{2}} \rho_{\Sigma} r_{\Sigma}^{2}$ & $r_{\Sigma}$ & $\frac{M}{M_{\odot}}$ & $Z_{0}$ & $Z_{\Sigma}$ \\
\hline 1 & 0.02 & 0.1182 & 05.635 & 0.076 & 0.03125 & 0.02062 \\
2 & 0.04 & 0.2342 & 07.931 & 0.214 & 0.06525 & 0.043062 \\
3 & 0.06 & 0.3437 & 09.658 & 0.391 & 0.10240 & 0.06600 \\
4 & 0.08 & 0.4572 & 11.081 & 0.598 & 0.14321 & 0.09109 \\
5 & 0.10 & 0.5690 & 12.362 & 0.834 & 0.18828 & 0.11803 \\
6 & 0.12 & 0.6687 & 13.401 & 1.085 & 0.23837 & 0.14707 \\
7 & 0.14 & 0.7690 & 14.371 & 1.358 & 0.29446 & 0.17851 \\
8 & 0.143 & 0.7831 & 14.502 & 1.399 & 0.30346 & 0.18345 \\
9 & 0.16 & 0.8649 & 15.241 & 1.646 & 0.35778 & 0.21267 \\
10 & 0.18 & 0.9568 & 16.030 & 2.035 & 0.42993 & 0.25000 \\
11 & 0.20 & 1.0452 & 16.754 & 2.262 & 0.51306 & 0.29100 \\
12 & 0.22 & 1.1254 & 17.385 & 2.581 & 0.61008 & 0.33630 \\
13 & 0.24 & 1.2066 & 18.001 & 2.916 & 0.72511 & 0.38541 \\
\hline 15 & 0.245 & 1.2252 & 18.140 & 3.000 & 0.75732 & 0.40045 \\
\hline & 0.25 & 1.2432 & 18.272 & 3.083 & 0.79113 & 0.41421 \\
\hline & & & & & &
\end{tabular}




\section{Conclusion}

We have given a new solution for spherically symmetric anisotropic fluid ball. It has been observed that the physical parameters pressure, density, and redshift are positive at the centre and within the limit of realistic state equation and monotonically decreasing and the causality condition is obeyed throughout the fluid ball. Thus, the solution is well behaved for all values of Schwarzschild parameter $S_{p}$ within the perfect fluid ball. Our solution is useful to construct the models of compact star like Strange star family, Neutron star and many more. We have discussed a model of massive neutron star having mass $2.95 M_{\odot}$ and radius $18 \mathrm{~km}$ with surface density $2 \times 10^{17} \mathrm{~kg} \cdot \mathrm{m}^{-3}$ and central density $3.1 \times 10^{17} \mathrm{~kg} \cdot \mathrm{m}^{-3}$. The central radial and tangential pressures of neutron star are $3.4 \times 10^{33} \mathrm{~N} / \mathrm{m}^{2}$ while the surface radial pressure of the star is zero and surface tangential pressure is $1.8 \times 10^{33} \mathrm{~N} / \mathrm{m}^{2}$. Table 1 shows that we can construct different models for neutron star having mass lies between $1.4 M_{\odot}$ and $3 M_{\odot}$ for different values of $S_{p}$ ranging from 0.143 to 0.245 . The solution reduces to Schwarzschild interior solution for $n=-1 / 2$ and for isotropic pressure.

\section{Acknowledgements}

Authors would like to thank to anonymous referee for rigorous review, constructive comments and valuable suggestions.

\section{References}

[1] Schwarzschild, K. (1916) On the Gravitational Field of a Mass Point According to Einstein's Theory. Sitzungsberichte der koniglich Preussischen Akademie der Wissenschaften Berlin (Mathematical Physics), 189-196.

[2] Tolman, R.C. (1939) Static Solutions of Einstein's Field Equations for Spheres of Fluid. Physical Review, 55, 364. https://doi.org/10.1103/PhysRev.55.364

[3] Buchdahl, H.A. (1959) General Relativistic Fluid Spheres. Physical Review D, 116, 1027. https://doi.org/10.1103/PhysRev.116.1027

[4] Delgaty, M.S.R. and Lake, K. (1998) Physical Acceptability of Isolated, Static, Spherically Symmetric, Perfect Fluid Solutions of Einstein's Equations. Computer Physics Communications, 115, 395. https://doi.org/10.1016/S0010-4655(98)00130-1

[5] Adler, R.J. (1974) A Fluid Sphere in General Relativity. Journal of Mathematical Physics, 15, 727. https://doi.org/10.1063/1.1666717

[6] Heintzmann, H.Z. (1969) Newtonian Time in General Relativity. Physik, 228, 489-493.

[7] Finch, N.R. and Skea, J.E.F. (1989) A Realistic Stellar Model Based on an Ansatz of Duorah and Ray. Class Quantum Gravity, 6, 467. https://doi.org/10.1088/0264-9381/6/4/007

[8] Patwardhan, G.K. and Vaidya, P.C. (1943) Relativistic Distributions of Matter of Radial Symmetry. Journal of the University of Bombay, 12, 23, Part III.

[9] Mehra, A.L. (1966) Radially Symmetric Distribution of Matter. Journal of the Australian Mathematical Society, 6, 153. https://doi.org/10.1017/S1446788700004730

[10] Kuchowicz, B. (1968) General Relativistic Fluid Spheres. II. Solutions of the Equation for $e^{-\lambda}$. Acta Physica Polonica, 34, 131 
[11] Matese, J.J. and Whitman, P.G. (1980) New Method for Extracting Static Equilibrium Configurations in General Relativity. Physical Review D, 22, 1270.

https://doi.org/10.1103/PhysRevD.22.1270

[12] Durgapal, M.C. (1982) A Class of New Exact Solutions in General Relativity. Journal of Physics A: Mathematical and General, 15, 2637. https://doi.org/10.1088/0305-4470/15/8/039

[13] Nariai, S. (1950) On Some Static Solutions of Einstein's Gravitational Field Equations in a Spherically Symmetric Case. Science Reports of the Tohoku University Series, 134, 160.

[14] Goldman, S.P. (1978) Physical Solutions to General-Relativistic Fluid Sphere. The Astrophysical Journal, 226, 1079. https://doi.org/10.1086/156684

[15] Ivanov, B.V. (2012) Collapsing Shear-Free Perfect Fluid Spheres with Heat Flow. General Relativity and Gravitation , 44, 1835-1855. https://doi.org/10.1007/s10714-012-1370-3

[16] Ivanov, B.V. (2002) Static Charged Perfect Fluid Spheres in General Relativity. Physical Review D, 65, Article ID: 104001. https://doi.org/10.1103/physrevd.65.104001

[17] Pant, N. (2011) Some New Exact Solutions with Finite Central Parameters and Uniform Radial Motion of Sound. Astrophysics and Space Science, 331, 633-644. https://doi.org/10.1007/s10509-010-0453-4

[18] Maurya, S.K. and Gupta, Y.K. (2013) Charged Fluid to Anisotropic Fluid Distribution in General Relativity. Astrophysics and Space Science, 344, 243-251. https://doi.org/10.1007/s10509-012-1302-4

[19] Pant, N., Fuloria, P. and Tewari, B.C. (2012) A New Well Behaved Exact Solution in General Relativity for Perfect Fluid. Astrophysics and Space Science, 340, 407-412. https://doi.org/10.1007/s10509-012-1068-8

[20] Pant, N., Fuloria, P. and Pradhan, N. (2014) An Exact Solution of Perfect Fluid in Isotropic Coordinates, Compatible with Relativistic Modeling of Star. International Journal of Theoretical Physics, 53, 993-1002. https://doi.org/10.1007/s10773-013-1892-9

[21] Herrera, L., Santos, N.O. and Wang, A. (2008) Shearing Expansion-Free Spherical Anisotropic Fluid Evolution. Physical Review D, 78, Article ID: 084026.

[22] Herrera, L., Ospino, J. and Perisco, A.D. (2008) All Static Spherically Symmetric Anisotropic Solutions of Einstein's Equations. Physical Review D, 77, Article ID: 027502. https://doi.org/10.1103/PhysRevD.77.027502

[23] Komathiraj, K. and Maharaj, S.D. (2007) Tikekar Superdense Stars in Electric Fields. Journal of Mathematical Physics, 48, Article ID: 042501. https://doi.org/10.1063/1.2716204

[24] Thirukkanesh, S. and Regel, F.C. (2012) Exact Anisotropic Sphere with Polytropic Equation of State. Pramana-Journal of Physics, 78, 687-696.

[25] Sunzu, J.M., Maharaj, S.D. and Ray, S. (2014) Charged Anisotropic Models for Quark Stars. Astrophysics and Space Science, 352, 719-727. https://doi.org/10.1007/s10509-014-1918-7

[26] Chaisi, M. and Maharaj, S.D. (2006) Anisotropic Static Solutions in Modelling Highly Compact Bodies. Pramana-Journal of Physics, 66, 609. https://doi.org/10.1007/BF02704504

[27] Maurya, S.K. and Gupta, Y.K. (2012) A Family of Anisotropic Super-Dense Star Models Using a Space-Time Describing Charged Perfect Fluid Distributions. Physica Scripta, 86, Article ID: 025009. https://doi.org/10.1088/0031-8949/86/02/025009

[28] Gupta, Y.K. and Maurya, S.K. (2011) A Class of Regular and Well Behaved Relativistic Super-Dense Star Models. Astrophysics and Space Science, 332, 155-162. https://doi.org/10.1007/s10509-010-0503-y 
[29] Fuloria, P., Tewari, B.C. and Joshi, B.C. (2011) Well Behaved Class of Charge Analogue of Durgapal's Relativistic Exact Solution. Journal of Modern Physics, 2, 1156-1160. https://doi.org/10.4236/jmp.2011.210143

[30] Whitman, P.G. and Burch, R.C. (1981) Charged Spheres in General Relativity. Physical Review D, 24, 2049. https://doi.org/10.1103/PhysRevD.24.2049

[31] Bonner, W.B. and Vickers, P.A. (1981) Junction Conditions in General Relativity. General Relativity and Gravitation, 13, 29-36.

[32] Pant, N. and Negi, P.S. (2012) Variety of Well Behaved Exact Solutions of Einstein-Maxwell Field Equations: An Application to Strange Quark Stars, Neutron Stars and Pulsars. Astrophysics and Space Science, 338, 163-169. https://doi.org/10.1007/s10509-011-0919-z

[33] Herrera, L. and Santos, N.O. (1997) Local Anisotropy in Self-Gravitating Systems. Physics Reports, 286, 53-130. https://doi.org/10.1016/S0370-1573(96)00042-7

[34] Tikekar, R. (1984) Spherical Charged Fluid Distributions in General Relativity. Journal of Mathematical Physics, 25, 1481-1483. https://doi.org/10.1063/1.526318

[35] Gupta, Y.K. and Kumar, M. (2005) A Superdense Star Model as Charged Analogue of Schwarzschild's Interior Solution. General Relativity and Gravitation, 37, 575-583. https://doi.org/10.1007/s10714-005-0043-x

[36] Herrera, L., Ospino, J., Di Parisco, A., Fuenmayor, E. and Triconis, O. (2009) Structure and Evolution of Self-Gravitating Objects and the Orthogonal Splitting of the Riemann Tensor. Physical Review D, 79, Article ID: 064025. https://doi.org/10.1103/PhysRevD.79.064025

[37] Herrera, L., Di Parisco, A., Hernandez, P. and Santos, N.O. (2004) Dynamics of Dissipative Gravitational Collapse. Physical Review D, 70, Article ID: 084004. https://doi.org/10.1103/PhysRevD.70.084004

[38] Herrera, L., Di Parisco, A., Hernandez, P. and Santos, N.O. (1998) On the Role of Density Inhomogeneity and Local Anisotropy in the Fate of Spherical Collapse. Physics Letters A, 237, 113-118. https://doi.org/10.1016/S0375-9601(97)00874-8

[39] Herrera, L., Santos, N.O. andWang, A.Z. (2008) Shearing Expansion-Free Spherical Anisotropic Fluid Evolution. Physical Review D, 78, 084026. http://dx.doi.org/10.1103/PhysRevD.78.084026

[40] Tewari, B.C. and Charan, K. (2015) Horizon Free Eternally Collapsing Anisotropic Radiating Star. Astrophysics and Space Science, 357, 107. https://doi.org/10.1007/s10509-015-2335-2

[41] Tewari, B.C. and Charan, K. (2014) Radiating Star, Shear-Free Gravitational Collapse without Horizon. Astrophysics and Space Science, 351, 613-617. https://doi.org/10.1007/s10509-014-1851-9

[42] Tewari, B.C., Charan, K. and Rani, J. (2016) Spherical Gravitational Collapse of Anisotropic Radiating Fluid Sphere. International Journal of Astronomy and Astrophysics, 6, 155-165.

[43] Tewari, B.C. (2013) Collapsing Shear-Free Radiating Fluid Spheres. General Relativity and Gravitation, 45, 1547-1558. https://doi.org/10.1007/s10714-013-1545-6

[44] Ivanov, B.V. (2010) Evolving Spheres of Shear-Free Anisotropic Fluid. International Journal of Modern Physics A, 25, 3975-3991.

[45] Ivanov, B.V. (2010) Evolving Spheres of Shear-Free Anisotropic Fluid. International Journal of Modern Physics A, 25, 3975-3991. https://doi.org/10.1142/S0217751X10050202

[46] Ivanov, B.V. (2011) Self-Gravitating Spheres of Anisotropic Fluid in Geodesic Flow. International Journal of Modern Physics D, 20, 319-334.

https://doi.org/10.1142/S0218271811018858 
[47] Ivanov, B.V. (2016) A Different Approach to Anisotropic Spherical Collapse with Shear and Heat Radiation. International Journal of Modern Physics D, 25, Article ID: 1650049. https://doi.org/10.1142/S0218271816500498

[48] Ivanov, B.V. (2016) All Solutions for Geodesic Anisotropic Spherical Collapse with Shear and Heat Radiation. Astrophysics and Space Science, 361, 18.

https://doi.org/10.1007/s10509-015-2603-1

[49] Maurya, S.K. and Gupta, Y.K. (2014) A New Class of Relativistic Charged Anisotropic Super Dense Star Models. Astrophysics and Space Science, 353, 657-665.

[50] Pant, N., Mehta, R.N., Pant, M.J. (2010) New Class of Regular and Well Behaved Exact Solutions in General Relativity. Astrophysics and Space Science, 330, 353-359. https://doi.org/10.1007/s10509-010-0383-1

[51] Pant, et al. (2011) Well Behaved Parametric Class of Exact Solutions of Einstein-Maxwell Field Equations in General Relativity. Journal of Modern Physics, 2, 1538-1543. https://doi.org/10.4236/jmp.2011.212186

\section{Submit or recommend next manuscript to SCIRP and we will provide best service for you:}

Accepting pre-submission inquiries through Email, Facebook, LinkedIn, Twitter, etc. A wide selection of journals (inclusive of 9 subjects, more than 200 journals)

Providing 24-hour high-quality service

User-friendly online submission system

Fair and swift peer-review system

Efficient typesetting and proofreading procedure

Display of the result of downloads and visits, as well as the number of cited articles Maximum dissemination of your research work

Submit your manuscript at: http://papersubmission.scirp.org/

Or contact wjm@scirp.org 